\title{
Personality traits and coping styles were associated with depression in spinal cord injury
}

\author{
Dias de Carvalho SA, Andrade MJ, Tavares MA, et al. Spinal cord injury and psychological response. Gen Hosp Psychiatry 1998 \\ Nov;20:353-9.
}

\section{Question}

In patients who have spinal cord injury (SCI), what is the relation between psychopathological response and personality traits, coping styles, and type of SCI?

\section{Design}

Cohort assembled within 6 weeks of admission to a rehabilitation unit with up to 78 weeks of follow up.

\section{Setting}

A rehabilitation unit at a hospital in Oporto, Portugal.

\section{Patients}

65 patients who were 18-75 years of age (mean age 39 y, $74 \%$ men) and were admitted to treatment in a rehabilitation unit (mean inpatient stay 24 weeks [range 3-78 weeks]). Exclusion criteria were concomitant head injury, malignancy, history of psychiatric disease, or age $<18$ years.

\section{Assessment of prognostic factors}

Sociodemographic variables, somatic and psychopathological events, and family history were assessed by a questionnaire. The Symptom Check List-90-Revised (SCL-90-R) was used to assess somatisation, obsessive compulsiveness, interpersonal sensitivity, depression, anxiety, hostility, phobic anxiety, paranoid ideation, and psychoticism; the Total of Positive Symptoms was also reported. A Global Severity Index was obtained by combining the 9 SCL-90-R scales. Personality traits were assessed by the Eysenck Personality Inventory (EPI), and the Coping Styles Evaluation Scale was used to assess coping styles.

\section{Main outcome measure}

Psychopathology (assessed by the SCL-90-R). Depression was defined as a score $\geqslant 1$ on the SCL-90-R subscale for depression.

\section{Main results}

$57 \%(95 \%$ CI $44 \%$ to $69 \%$ ) of patients were depressed in the first 6 weeks, $40 \%$ (CI 28\% to 53\%) were depressed in the last 6 weeks, and 33\% (CI 21\% to $45 \%$ ) were persistently depressed at both assessments. EPI neuroticism was positively associated with depression in the first 6 weeks $(r=0.51, \mathrm{p}<0.001)$ and in the last 6 weeks $(r=0.39, \mathrm{p}<0.01)$. EPI extroversion was negatively associated with depression in the first 6 weeks $(r=-0.41, \mathrm{p}<0.001)$ and in the last 6 weeks $(r=-0.37, \mathrm{p}<0.01)$. Depression was positively associated with coping styles that used confrontation $(r=0.25$ to $r=0.32, \mathrm{p} \leqslant 0.05)$, escape or avoidance $(r=0.28$, $\mathrm{p}<0.05)$, and self blame $(r=0.30, \mathrm{p}<0.01)$. Coping styles that were negatively associated with depression were acceptance $(r=-0.26, \mathrm{p}<0.05)$, distancing $(r=-0.31, \mathrm{p}<0.01)$, and self control $(r=-0.24, \mathrm{p}<0.05)$. No differences existed between quadriplegic and paraplegic patients for depression.

\section{Conclusions}

In patients with spinal cord injury, 33\% were persistently depressed at follow up. Depression was positively associated with coping styles that used confrontation, escape or avoidance, and self blame, and was negatively associated with acceptance, distancing, and self control. No differences existed between quadriplegic and paraplegic patients for depression.

Source of funding: no external funding.

For correspondence: Dr S A Dias de Carvalho, R Prof Correia de Araújo 617-5.1, 4200 Porto, Portugal. Fax +35125027018.

\section{Commentary}

This study by Dias de Carvalho et al examines the prevalence of depression and associated coping styles in a Portuguese sample of people with SCI. Similar studies have been done in the US and the UK. ${ }^{2}$ All of these studies have highlighted the relation between adjustment after SCI and ways of coping. In this study, however, the prevalence of depression in the acute stage is higher than in other studies. The authors have used the SCL-90-R as an outcome measure and defined depression as a score of $\geqslant 1$ on the depression subscale. Hanson et al also used this measure but did not report subscale results, presumably because of the high internal consistency of the scale. ${ }^{1}$

Much of the extant literature has used self report measures, which have been found to be valid. ${ }^{3}$ Estimates of the prevalence of depression with this population range from $30 \%$ to $60 \%$. The prevalence of depression after SCI is important to examine because it is often associated with the occurrence of secondary complications, such as pressure sores, urinary tract infections, and contractures. This study confirms the need to address depression within this population.

With respect to the conclusions on coping styles and the prevalence of depression, the patterns mentioned in this study are similar to the findings of other studies, but they are only of a correlational nature. It is difficult therefore to be certain about the degree of causality between coping strategies and mood state. This question would be more appropriately addressed by a detailed longitudinal methodology that explores such relations from the acute phase to years after discharge.

Paul Kennedy, DPhil, MSc, FBPS Stoke Mandeville Hospital NHS Trust Aylesbury, UK

1 Hanson S, Buckelew SP, Hewett J, et al. The relationship between coping and adjustment after spinal cord injury: a 5-year follow-up study. Rehabilitation Psychology 1993;38:41-52. 2 Kennedy P, Lowe R, Grey N, et al. Traumatic spinal cord injury and psychological impact: a cross-sectional analysis of coping strategies. $\mathrm{Br}$ Clin Psychol 1995;34:627-39.

3 Elliot TR, Frank RG. Depression following spinal cord injury. Arch Phys Med Rehabil 1996;77: 816-23. 